\title{
Komplementärmedizin: Spreu vom Weizen trennen
}

\section{Komplementärmethoden in der Allergologie sollten nicht zwangs- läufig als Hokuspokus abqualifiziert werden. Allerdings erfreuen sich heute einige Therapieformen bei den Patienten großer Beliebtheit, von denen der seriös arbeitende Arzt abraten sollte.}

In der allergologischen Schulmedizin stiefmütterlich behandelte sogenannte „Alternative Heilmethoden“ müssen nicht immer unseriös sein. Hier die Spreu vom Weizen zu trennen hat sich der Arbeitskreis ,Komplementärmedizin“ der DGAI zur Aufgabe gestellt. Diesem Arbeitskreis gehören u.a. namhafte Vertreter der Allergologie, Phytotherapie, Balneologie und anderer Naturheilverfahren an.

Wie W. Dorsch (München) ausführte, hat dieser Arbeitskreis folgende Heilmethoden als seriös und damit als für die schulmedizinische allergologische Begleitbehandlung geeignet eingestuft: Atemtherapie, Akupunktur, autogenes Training, Balneotherapie, funktionelle Entspannung, Klimatherapie, bestimmte Diäten sowie Phyto-, Physio- und Psychotherapie (Allergo Journal berichtet über einzelne dieser Methoden in den nächsten Ausgaben).

\section{Von Bioresonanz über Bachblüten bis zur Eigenbluttherapie}

Doch tummeln sich auf dem Markt der Alternativmethoden auch Behandlungsangebote, deren Wert keiner wissenschaftlichen Nachprüfung standhält und von denen der Arzt abraten sollte. Dazu gehört z.B. die Bioresonanzdiagnostik, bei der versucht wird, durch Veränderungen von elektrischer Spannung den Auslöser der Allergie dingfest zu machen. Diese Veränderungen

\section{Alternativmethoden - bei den Patienten hoch im Kurs}

Der Leidensdruck von Allergiepatienten ist offenbar sehr groß. Immer häufiger suchen diese Patienten ihr Heil in teilweise zweifelhaften Alternativmethoden, wobei rund die Hälfte pro Therapieversuch mehr als 1000 DM aus eigener Tasche bezahlen. Dies ist ein Ergebnis einer Untersuchung des Deutschen Allergie- und Asthmabundes (DAAB).

So gaben immerhin $61 \%$ von 360 Befragten an, bereits 2 bis 4 alternative Heilverfahren angewendet zu haben. Jeder fünfte hat sogar schon mehr als 5 verschiedene Methoden ausprobiert - mit unterschiedlichem Erfolg: Über gute und lang anhaltende Therapieerfolge mit Homöopathie und Akupunktur berichteten rund ein Drittel der Patienten. Einer Eigenblutbehandlung hatten sich rund 40\% (!) der Befragten schon einmal unterzogen, ihre Wirksamkeit wurde von knapp 60\% der Patienten als ,wenig oder nicht erfolgreich“ bewertet. Etwas schlechter schnitten die Bachblütentherapie mit $62 \%$ und Handauflegen mit 75\% Enttäuschten ab. Zahlen in vergleichbarer Größenordnung ergaben sich auch in einer Studie von T. Schäfer et al. (Allergo Journal, Heft 7/99, S. 301-304).

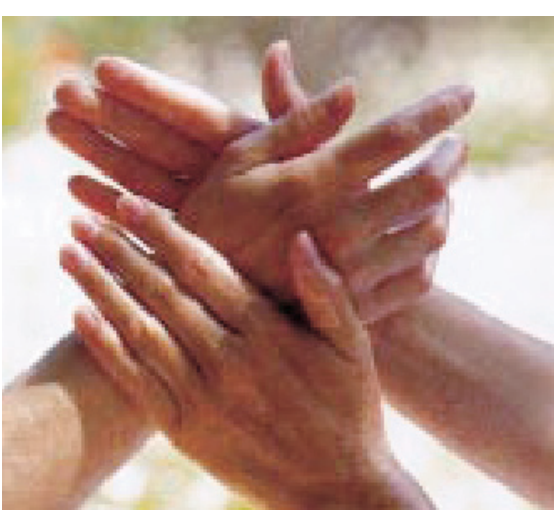

Ob Geistheilung oder Bioresonanztherapie - bei vielen Alternativverfahren fühlt sich der Patient offenbar besser umsorgt als beim Allergologen, der Markt für diese wissenschaftlich nicht anerkannten Heilmethoden boomt jedenfalls.

sollen sich dann ergeben, wenn der $\mathrm{Pa}$ tient mit einem in Glasampullen abgefüllten Allergen in Berührung kommt.

Als Unfug, wenn nicht gar Betrug muß nach Dorsch die Bioresonanztherapie gewertet werden, die von sich behauptet, mit einer speziellen Technik negative elektrische Impulse, die z.B. durch Allergien verursacht werden, durch positive neutralisieren zu können und Allergien damit zu ,löschen“.

Ebenfalls in Mode ist die Autohomologe Immuntherapie, bei der Patientenblut, manchmal auch -urin, biochemisch nach einem Geheimrezept verändert und dem Patienten anschließend auf oralem, nasalem, inhalativen und/oder parenteralem Weg wieder appliziert wird.

Bei der Eigenblutbehandlung wird Vollblut venös entnommen und intramuskulär injiziert. Durch diesen unspezifischen Reiz soll es zu einer günstigen Wirkung auf die Erkrankung kommen. Verläßliche Belege für diese Behauptung in der Allergologie und Pneumologie fehlen jedoch, so Dorsch.

Einen negativen Wirksamkeitsbescheid erhält auch das ebenfalls zur Zeit sehr beliebte Verfahren der Bachblütentherapie, bei der Blüten bestimmter Pflanzen mit Quellwasser über Nacht mazeriert werden und das Wasser anschließend weiter verarbeitet oder getrunken wird.

$b k$

W. Dorsch (München) anläßlich der 21. Tagung der Deutschen Gesellschaft für Allergologie und klinische Immunologie, München 1999. 